\title{
Depresión: estado del conocimiento y la necesidad de políticas públicas y planes de acción en México
}

\author{
Shoshana Berenzon, D en Psic, (I) María Asunción Lara, D en CS,, ${ }^{(1)}$ Rebeca Robles, D en Psic, (I) \\ María Elena Medina-Mora, D en Psic.(I)
}

\begin{abstract}
Berenzon S, Lara MA, Robles R, Medina-Mora ME. Depresión: estado del conocimiento y la necesidad de políticas públicas y planes de acción en México. Salud Publica Mex 2013;55:74-80.
\end{abstract}

\begin{abstract}
Resumen
La depresión constituye un problema importante de salud pública. En el mundo, representa la cuarta causa de discapacidad en cuanto a la pérdida de años de vida saludables. En México ocupa el primer lugar de discapacidad para las mujeres y el noveno para los hombres. La depresión tiene una alta co-ocurrencia con otros trastornos como la ansiedad, el consumo de sustancias, la diabetes y las enfermedades cardiacas. Pese al impacto que tiene este trastorno, una gran proporción de personas no acude a tratamiento, retrasa mucho la búsqueda de ayuda, o bien no recibe la asistencia adecuada. El propósito de este ensayo es exponer el resultado de una breve revisión de investigaciones que permiten ubicar la depresión como un problema de salud pública en México. Se abordan la prevalencia y los factores asociados, las brechas de atención, el uso de servicios y los tratamientos disponibles. Al final se plantean algunas de las implicaciones para la investigación y para las políticas públicas.
\end{abstract}

Palabras clave: depresión; atención al paciente; salud pública; México
Berenzon S, Lara MA, Robles R, Medina-Mora ME.

Depression: state of the art and the need for public policy and action plans in Mexico.

Salud Publica Mex 2013;55:74-80.

\section{Abstract}

Depression is an important public health problem. It is the fourth cause of disease in the world in terms of lost years of healthy life. In Mexico, it ranks first in terms disability for women and ninth for men. There is a high comorbidity between depression and other mental disorders such as anxiety and substance abuse, as well as other serious and chronic physical conditions (e.g. diabetes, and heart disease). Despite the impact of depressive disorders in the quality of life of the population, there is a large proportion of people who don't get treatment, delaying seeking help and thus don't receive adequate assistance. The aim of this paper is to present an analysis of depression status in the Mexican population from a public health perspective; it includes prevalence and associated factors, gaps in care, characteristics of the use of services and treatments available. The paper concludes with a presentation of the implications for research and mental health policy in Mexico.

Key words: depression; patient care; public health; Mexico
L a depresión contribuye de manera significativa a la $ـ$ carga global de enfermedad; 1 es una de las principales causas de discapacidad y se estima que afecta a 350 millones de personas en el mundo, con prevalencias que oscilan entre 3.3 y $21.4 \% .^{2}$ Este trastorno generalmente comienza en edades tempranas, reduce sustancialmente el funcionamiento de las personas, es un padecimiento recurrente y tiene importantes costos económicos y sociales. Es por ello que la depresión se encuentra entre los primeros lugares de la lista de enfermedades discapacitantes y se ha convertido en un objetivo prioritario de atención en todo el mundo. Así entonces, el objetivo de este trabajo es presentar una breve revisión de investigaciones actuales que permiten ubicar a la depresión

(I) Instituto Nacional de Psiquiatría Ramón de la Fuente Muñiz México. DF. México.

\footnotetext{
Fecha de recibido: 28 de mayo de 2012 - Fecha de aceptado: 10 de octubre de 2012

Autor de correspondencia: Shoshana Berenzon Gorn. Instituto Nacional de Psiquiatría. Calzada México-Xochimilco I0I. Delegación Tlalpan I4370 México DF, México correo electrónico: berenz@imp.edu.mx
} 
como un problema importante de salud pública en México. Se abordan los factores asociados y las tasas de prevalencia, las brechas de atención, las características del uso de servicios y los tratamientos disponibles. El trabajo concluye con la presentación de las implicaciones para la investigación y para las políticas públicas.

\section{Factores psicosociales asociados con la depresión}

Hoy en día existe acuerdo al señalar que la depresión, como la mayoría de las enfermedades, es el resultado de la interacción entre factores biológicos, heredados, del desarrollo y del contexto, que interactúan con las características individuales. Por ende, su estudio requiere de múltiples aproximaciones. Sin dejar de reconocer la importancia de analizar los avances en el campo de las neurociencias y la genética, que han proporcionado bases para corregir la división mente-cuerpo que constituyó una barrera para entender la relación entre cerebro, pensamiento, emoción y conducta, el presente trabajo se enfocará en el análisis de los factores psicosociales asociados con la depresión que pueden tener implicaciones para las políticas públicas.

En población mexicana, entre las variables psicosociales asociadas con la depresión destacan: a) ser mujer (más aún si se es jefa de familia), dedicarse exclusivamente a las labores del hogar y si se ha adquirido la responsabilidad de cuidar a algún enfermo; b) tener un bajo nivel socioeconómico (por la mayor exposición a las vicisitudes de la pobreza); c) estar desempleado (sobre todo en los hombres); d) el aislamiento social; e) tener problemas legales; $\mathrm{f}$ ) tener experiencias de violencia; $\mathrm{g}$ ) consumir sustancias adictivas, y h) la migración. ${ }^{3-7}$

Factores del entorno como la violencia, la inseguridad o las crisis económicas incrementan el riesgo de que se presente la sintomatología depresiva. Los datos de la Encuesta Nacional de Epidemiología Psiquiátrica (ENEP) muestran que una proporción importante de la población nacional ha estado expuesta a eventos violentos y que éstos tienen importantes variaciones por género. Las mujeres presentan prevalencias más elevadas de abuso sexual, violencia física por parte de la pareja, violación, persecución y acoso. Por su parte, los hombres experimentaron más atracos con arma y accidentes; también fueron testigos, en mayor proporción que las mujeres, de muerte o lesiones a una persona, sufrieron más secuestros y mataron accidentalmente a otro en un porcentaje mayor. Una proporción de estas personas desarrolló estrés post traumático $(2.3 \%$ de las mujeres y $0.5 \%$ de los hombres) y alrededor de $20 \%$ de personas que han sufrido uno o más eventos violentos, presentaron un cuadro de depresión. ${ }^{8}$
Las constantes crisis económicas y sociales que se han vivido en México han tenido como consecuencia que, entre las principales fuentes de preocupación cotidiana, se encuentren las relacionadas con problemas financieros, y que términos como "sufrimiento" y "tristeza" sean muy utilizados para dar cuenta de este tipo de preocupaciones. En este sentido, Medina-Mora y colaboradores ${ }^{9}$ señalan que si bien no hay diferencias significativas por nivel socioeconómico en la prevalencia de trastornos depresivos, la población que vive en condiciones de pobreza presenta una depresión más grave que aquélla que se encuentra en mejores condiciones económicas.

\section{Características de la depresión en México}

Los resultados de la ENEP señalan que 9.2\% de los mexicanos sufrieron un trastorno afectivo en algún momento de su vida y $4.8 \%$ en los doce meses previos al estudio. La depresión ocurre con mayor frecuencia entre las mujeres $\left(10.4 \%\right.$ ) que entre los hombres $(5.4 \%) .^{5}$ Las prevalencias observadas en los EUA, Europa y Brasil son más elevadas ya que más de $15 \%$ de la población ha padecido depresión mayor alguna vez en la vida y más de $6 \%$ durante el año anterior. ${ }^{10,11}$ En países como Colombia y Chile, la situación es muy similar a la de México. ${ }^{12,13}$

Como ya se mencionó, los trastornos depresivos producen importantes niveles de discapacidad, afectan las funciones físicas, mentales y sociales y se asocian con mayor riesgo de muerte prematura. Pueden dificultar la capacidad de la persona para realizar actividades cotidianas, causando un deterioro significativo en el funcionamiento habitual y en la calidad de vida. Los análisis recientes realizados en México muestran que la depresión produce mayor discapacidad que otras condiciones crónicas como la diabetes, los trastornos respiratorios, las enfermedades cardiacas o la artritis. Por ejemplo, las personas con depresión pierden hasta 2.7 más días de trabajo que aquellas con otro tipo de enfermedades crónicas. ${ }^{14}$

De este modo, se considera que la depresión es una de las principales causas de carga de enfermedad en términos de años de vida ajustados por discapacidad. La Organización Mundial de la Salud (OMS) prevé que en el año 2020 sea la segunda causa de discapacidad en el mundo, tan sólo después de las patologías cardiovasculares. ${ }^{1}$ En México, es la principal causa de pérdida de años de vida ajustados por discapacidad para las mujeres y la novena para los hombres. ${ }^{15}$

La edad de inicio de la mayoría de los trastornos psiquiátricos se ubica en las primeras décadas de la vida; $50 \%$ de los adultos que han sufrido un trastorno 
mental lo padecieron antes de cumplir 21 años. Cuando los trastornos depresivos se presentan antes de los 18 años, el curso de la enfermedad es más crónico y de mayor duración. 5,16

Estudios con población adolescente muestran que, entre los trastornos más frecuentes en esa etapa de la vida, se encuentran los depresivos; $7.8 \%$ de los jóvenes cumplieron con los criterios diagnósticos de depresión mayor y $0.8 \%$ con aquéllos de distimia. Estos padecimientos generan discapacidad entre los adolescentes debido a que pueden interferir en su desarrollo y limitarlos en la toma de decisiones importantes en su vida. Es probable que la presencia de síntomas depresivos se relacione con el maltrato, la falta de oportunidades para realizar sus aspiraciones y la violencia del entorno en que viven, entre otros factores. ${ }^{16}$

Entre quienes tienen más de 65 años, la prevalencia de depresión se encuentra arriba de $20 \%$ y es más frecuente entre los desempleados, los viudos y los solteros. ${ }^{17}$ En esta población, los problemas mentales son una de las cinco principales causas de discapacidad. ${ }^{18}$ En México, la transición hacia una población con mayor número de ancianos es una realidad. De acuerdo con la OMS, dentro de 25 años la población mayor de 60 años que vive en América Latina y el Caribe pasará de 40 millones a más de 97 millones. Resulta necesario pensar en estrategias que permitan enfrentar los trastornos mentales propios de este grupo etario.

Otro grupo que merece especial atención son las mujeres con depresión en etapa perinatal. La depresión posparto (DPP) no es muy diferente de la depresión en otro momento de la vida. De acuerdo con el Manual diagnóstico y estadístico de los trastornos mentales (DSM-IV), es similar a la depresión mayor pero con manifestaciones de ansiedad intensa, e incluso crisis de angustia, así como una preocupación excesiva por el bebé. Su importancia radica en que en este momento de la vida tiene consecuencias muy negativas sobre la salud y el desempeño de la madre y sobre el desarrollo cognitivo, conductual y emocional del infante. ${ }^{19}$ De 5 a $25 \%$ de las mujeres padecen $\mathrm{DPP}^{20}$ y se le considera la causa principal de enfermedad relacionada con discapacidad en mujeres, así como la complicación más común y más seria del parto. ${ }^{21}$ En México ha sido poco estudiada, pero datos recientes informan de una prevalencia de $32.6 \%$ (14.1\% depresión mayor y $18.5 \%$ depresión menor). ${ }^{22}$

Los principales factores de riesgo para DPP son: ansiedad prenatal, escaso apoyo social, eventos estresantes recientes, experiencias traumáticas, baja autoestima, conflictos de pareja, factores obstétricos, nivel socioeconómico, escolaridad y antecedentes familiares de depresión. 22,23
Es primordial enfatizar que la DPP debe ser tratada con intervenciones psicológicas, farmacológicas, o con ambas. Estos tratamientos han sido satisfactoriamente evaluados en esta población. ${ }^{23}$ La prevención de DPP durante el embarazo muestra resultados alentadores. En México, hay evidencia de que una intervención psicoeducativa grupal reduce la incidencia de DPP. ${ }^{24}$

Aunque la investigación y el cuidado clínico han puesto mayor énfasis en el periodo del posparto, la depresión durante el embarazo tiene considerable importancia; de 2 a $21 \%$ de las gestantes -y con sintomatología depresiva, de 8 a $31 \%-{ }^{25}$ la padecen. En México, estos síntomas se observan en 22 a $37 \%$ de las gestantes. ${ }^{26}$ La depresión no tratada durante el embarazo también tiene consecuencias adversas que se asocian con un incremento en la tasa de complicaciones obstétricas, óbitos, intentos de suicidio, cuidados posparto especializados para el infante, productos con bajo peso al nacer y retraso en el desarrollo. Los principales factores de riesgo de depresión durante la gestación son embarazo no planeado, ser madre soltera, ideación suicida, no ser el primer embarazo, falta de apoyo social y trastorno depresivo previo. ${ }^{23}$

\section{Comorbilidad de la depresión con otras enfermedades crónicas}

La relación entre las enfermedades mentales y físicas es muy estrecha. Para la mayoría de las personas resulta difícil y preocupante aprender a sobrellevar y vivir con una dolencia crónica de larga duración como la diabetes o el cáncer, por lo que no es sorprendente que la depresión sea la complicación más común asociada con este tipo condiciones. ${ }^{27}$

La investigación demuestra que las personas con enfermedades físicas graves corren un mayor riesgo de padecer depresión. En las personas con diabetes el riesgo de presentar depresión es dos veces más alto que en aquellas que no sufren esta condición; para la población con asma u obesidad, el riesgo aumenta tres veces y para aquellas que sufren de dolores crónicos el riesgo crece cinco veces. ${ }^{6}$ Del mismo modo, las personas que padecen síntomas de depresión tienen mayores probabilidades de presentar enfermedades cardiovasculares, diabetes, cáncer y enfermedades respiratorias, todas ellas condiciones crónicas responsables de más de $60 \%$ de las muertes en el mundo. ${ }^{28}$

Asimismo es frecuente una alta comorbilidad entre la depresión y otras patologías mentales, como los trastornos de ansiedad y el consumo de sustancias adictivas. ${ }^{29}$ La literatura internacional indica que alrededor de la mitad de la población adulta con trastorno depresivo 
mayor cumple con criterios diagnósticos para uno o más trastornos de ansiedad; esta relación ocurre entre 25 y 50\% de los adolescentes con depresión y entre 10 y $15 \%$ de los jóvenes con ansiedad. ${ }^{30}$ Los estudios epidemiológicos realizados en México señalan que alrededor de 32\% de la población diagnosticada con un trastorno de consumo de sustancias adictivas (alcohol o drogas) presenta también un trastorno afectivo. Si se considera solamente a las personas con abuso o dependencia a sustancias ilícitas, la comorbilidad es mayor a 50\%. ${ }^{6}$

\section{Estrategias de atención}

Los avances en el conocimiento sobre la depresión no dejan duda respecto al tremendo impacto que ésta tiene en la calidad de vida de la población. Pese a ello, una gran mayoría de los individuos no acude a tratamiento, lo que conlleva a un incremento en la discapacidad y en el impacto económico y social.

En México, menos de 20\% de quienes presentan un trastorno afectivo buscan algún tipo de ayuda, ${ }^{9} \mathrm{y}$ se estima que quienes lo hacen tardan hasta 14 años en llegar a un tratamiento especializado. En este sentido, México es una de las naciones que muestra mayor retraso en la búsqueda de atención. ${ }^{31}$ Aunado a lo anterior, cuando los pacientes acceden a los servicios de salud, solamente $50 \%$ de ellos reciben algún tipo de tratamiento mínimo adecuado, es decir, de por lo menos cuatro sesiones de psicoterapia y, en el caso de prescripción de psicotrópicos, al menos dos visitas con el médico o psiquiatra y tratamiento con los fármacos por algún periodo. ${ }^{32}$

Diversos factores se asocian con la reducida solicitud de ayuda, tales como el estigma, los horarios de atención poco convenientes, las largas listas de espera para obtener una cita, el diferimiento de las consultas, el hecho de no contar con alguien que cuide a los hijos mientras se asiste a consulta, el desconocimiento del tratamiento o las dificultades para el trasladarse al servicio, entre otros. Un factor más tiene que ver con la falta de coincidencia entre las necesidades sentidas de la población y el tipo de asistencia que se ofrece; es decir, mientras la población suele definir y expresar las necesidades en términos de problemas cotidianos que tienen como consecuencia la presencia de estrés o tristeza (laborales, económicos, con la pareja, la familia, etc.), lo que ameritaría consejo, entendimiento o empatía, la atención de los servicios está basada en realizar diagnósticos psiquiátricos y dirige el tratamiento hacia los trastornos detectados. Esta falta de coincidencia en la manera de vivir y entender la enfermedad también dificulta que la población acuda y permanezca en los servicios. ${ }^{33}$
Como plantean Wagner y colaboradores, ${ }^{34}$ resulta necesario analizar con mayor detenimiento los significados y representaciones que las personas tienen en torno a la depresión y hacia los factores que facilitan $u$ obstaculizan el acceso a los servicios.

\section{Costoefectividad de los}

tratamientos para la depresión

La estimación de costoefectividad de intervenciones para un padecimiento permite conocer cuáles son los tratamientos que conviene gestionar, tomando en cuenta que los recursos disponibles para atender la demanda y necesidad en salud son finitos. Es a partir de este tipo de evidencia científica que los recursos disponibles (o potencialmente disponibles) pueden asignarse de forma óptima.

La OMS seleccionó los años de vida ajustados por discapacidad o AVISA (-en inglés DALYs, Disability Adjusted Life Years-) como las unidades de evaluación de costoefectividad de las intervenciones para diferentes problemas de salud, incluyendo los trastornos mentales como la depresión. ${ }^{35}$ Cuando se llevan a cabo estudios de costoefectividad tomando en cuenta esta unidad básica de medición, se calcula el costo que tendrían los AVISA evitados por cada intervención.

Es claro que en México las intervenciones disponibles para el tratamiento de la depresión en el primer nivel de atención evitarían un número considerable de AVISA; llaman la atención dos tipos de intervenciones, una con sólo medicamento y otra con la combinación de estrategias farmacológicas y psicosociales: a) el número de AVISAs evitados sería casi tres veces superior si se administraran antidepresivos tricíclicos con una cobertura de $50 \%$, y b) llegaría a ser hasta seis veces superior si se emplearan inhibidores de recaptura de serotonina más psicoterapia y manejo proactivo de caso. ${ }^{36}$

Este importante número de AVISA evitados con la intervención combinada, sugiere que la inclusión de tratamientos psicosociales es ventajosa desde el punto de vista de la relación costoefectividad. Afortunadamente, aunque hace falta invertir en más y mejores esfuerzos de investigación clínica para el desarrollo, adaptación y evaluación de tratamientos para la población nacional, se dispone de alguna evidencia de efectividad de los mismos.

\section{Efectividad de tratamientos para la depresión en población mexicana}

En el plano mundial se ha planteado que el tratamiento más adecuado para la depresión es aquel que combina 
el uso de fármacos antidepresivos con psicoterapia cognitivo conductual o interpersonal..$^{37}$ Existen diversas intervenciones psicológicas breves que han sido adaptadas con éxito para su empleo en muy diversas culturas y países ${ }^{38}$ y una ventaja de las mismas es que pueden ser reproducidas con efectividad por parte de personal que labora en el primer nivel de atención a la salud, con lo que la atención a la depresión no tiene porque encarecerse al ser otorgada por un especialista. En México, Páez y colaboradores ${ }^{39}$ demostraron que una intervención cognitivo conductual otorgada por un pasante de medicina presenta efectos significativos para cambios en tiempo y por grupo experimental (GE) y control (GC) (F=22.7, gl=1,50, $p=<0.001 ; \mathrm{F}=5.5, \mathrm{Gl}=1,50$, $p=0.02$ ) de sintomatología depresiva basal y a los cinco meses $(\mathrm{GE}=20.8 \pm 9.8$ y $11.0 \pm 10.3$, respectivamente; $\mathrm{GC}=$ $21.2 \pm 11.6$ y $17.9 \pm 13.9)$.

Una intervención psicoeducativa grupal para mujeres con elevada sintomatología depresiva también mostró reducción de los síntomas y gran aceptación por parte de las participantes. Esta intervención está siendo diseminada por el Instituto Nacional de Psiquiatría a personal especializado y no especializado en salud mental. ${ }^{40}$

Finalmente, se ha demostrado que estas intervenciones aportan beneficios a la población mexicana con padecimientos crónicos con alta comorbilidad de depresión, como es el caso de pacientes con diabetes. ${ }^{41}$ El tratamiento de la sintomatología depresiva se asoció, además, con la mejoría de la condición médica, lo que demuestra la necesidad de la atención integral a la salud; sin salud mental no hay salud física.

\section{Discusión}

La anterior revisión muestra cómo la depresión es una enfermedad que tiene un impacto significativo en la calidad de vida y en el funcionamiento cotidiano de la población. Es frecuente la comorbilidad con condiciones crónicas como la diabetes y las enfermedades coronarias, lo cual aumenta el impacto económico para el paciente, su familia y la sociedad. Se observa que sólo una proporción pequeña de personas afectadas acuden a tratamiento y, quienes lo hacen, tardan mucho tiempo en decidirse a hacerlo; aunado a esto, sólo la mitad recibe un tratamiento mínimo adecuado. Sin embargo, resulta alentadora la existencia en México de tratamientos costoefectivos disponibles que pueden mejorar la salud y calidad de vida de las personas con depresión, si son recibidos de manera oportuna. A continuación se plantean algunas de las implicaciones para la investigación y para las políticas públicas que pueden deducirse a partir de la información presentada.

\section{Implicaciones para la investigación}

La mayoría de los resultados que se presentan en este artículo provienen de encuestas nacionales realizadas durante varios años, la última en 2002, lo que hace necesario llevar a cabo nuevos estudios que permitan continuar con la evaluación de las tendencias en la prevalencia, los determinantes, el impacto a la salud de los trastornos depresivos, los cambios en la brecha de tratamiento y las actuales necesidades de atención. Se detectó la ausencia de estudios longitudinales que permitan analizar la evolución de la depresión y su relación con factores psicosociales, genéticos y económicos, así como con otros determinantes ambientales. Del mismo modo, dados los escasos recursos con los que se cuenta para la atención de la salud mental, es primordial contar con evidencia que muestre que las intervenciones derivadas de la investigación básica, clínica y psicosocial son costoefectivas y responden a las necesidades de las personas que padecen este trastorno. Por otro lado, con el fin de acercar los servicios a la población, se requiere profundizar en la percepción que tienen las personas de este trastorno mental. Asimismo, es necesario ampliar las investigaciones orientadas a reducir el estigma y las enfocadas en la promoción de la salud y prevención de enfermedades.

\section{Implicaciones para las políticas públicas}

Es necesario reconocer que la salud mental está hoy en día incluida en la agenda de la salud pública y que se han logrado avances importantes como la integración del tratamiento farmacológico y psicoterapéutico de los principales trastornos mentales en el Catalogo Universal de Servicios de Salud (CAUSES) del Seguro Popular. Sin embargo, el desafío está lejos de haberse vencido; sigue siendo ancha la brecha entre lo que se necesita y lo que está disponible para reducir la carga de los desórdenes mentales en general, y de la depresión en particular. Ante este panorama, los retos para mejorar la atención son: a) continuar con el proceso de gestión para que los trastornos mentales sean consideradas como enfermedades crónicas con derecho a tratamiento y lograr así un acceso universal para la atención de estos padecimientos; $b$ ) Las políticas públicas deben incorporar de manera equilibrada acciones de prevención, promoción, tratamiento y rehabilitación. Es importante que estas acciones se realicen de manera coordinada con los diferentes sectores públicos para lograr un uso más eficiente de los recursos disponibles. Aunado a lo anterior, es necesario que se consideren las dimensiones biopsicosociales de la enfermedad, los elementos culturales del padecer y los requerimientos específicos por género o edad, para 
responder de una manera más eficaz a las necesidades sentidas por la población; c) Fortalecer los servicios de salud mental en el primer nivel de atención con intervenciones clave que combinen el tratamiento farmacológico con la psicoterapia breve. En otros países se ha visto que la atención primaria proporciona continuidad en el tratamiento porque los pacientes y el equipo se conocen, lo que redunda en la reducción de los costos totales de la atención sanitaria. Adicionalmente, es frecuente que los resultados obtenidos cuando las intervenciones se realizan en el primer nivel sean mejores que los que se logran en el nivel más especializado, porque la atención primaria está mejor equipada para tratar a los pacientes de forma integral; d) Es clara la necesidad de aumentar el financiamiento para la atención de la salud mental, pero también es necesario lograr una mejor distribución de los recursos, ya que 80\% de los mismos se consumen en la manutención de los hospitales psiquiátricos, lo que reduce las posibilidades de invertir en acciones para fortalecer la atención en el primer nivel; e) Es necesario desarrollar programas para atender a los grupos más vulnerables (niños, comunidades rurales, indígenas, población en extrema pobreza, etc.), que generalmente tienen un acceso muy limitado a los servicios de salud mental existentes; f) En cuanto a la depresión posparto, es necesario implementar medidas para informar a la población sobre este padecimiento y fomentar su detección oportuna. La instrumentación de estas acciones en servicios gineco-obstétricos, pediátricos y de atención primaria, permitirá reducir las consecuencias negativas y la discapacidad asociadas con este trastorno en dos generaciones: la madre y el infante.

Si bien es cierto que en México existe evidencia suficiente sobre las dimensiones y costos de la depresión y también sobre las posibles acciones para prevenirla y tratarla, es necesario redoblar los esfuerzos. Unicamente se podrá reducir la carga que representa este padecimiento si se mantiene un proceso continuo de evaluación de los programas y de las políticas públicas.

Declaración de conflicto de intereses. Los autores declararon no tener conflicto de intereses.

\section{Referencias}

I. Murray CJL López, AD. The global burden of disease. A comprehensive assessment of mortality and disability from disease, injuries, and risk factors in 1990 and projected to 2020. Harvard University Press, World Health Organization and The World Bank, 1996.

2. Kessler RC, Angermeyer M, Anthony JC, De Graaf R, Demyttenaere $\mathrm{K}$, Gasquet I, et al. Lifetime prevalence and age-of-onset distributions of mental disorders in the World Health Organization's World Mental Health Survey Initiative. World Psychiatry 2007; 6 (3): I68-176.
3. Aguilar-Gaxiola S, Kramer E, Resendez C, Magaña C. The context of depression in Latinos in the United States. En: Aguilar- Gaxiola S, Gullotta $\mathrm{T}$, ed. Depression in Latinos. Assessment, treatment and prevention. New York: Springer, 2008.

4. Fleiz C, Ito E, Medina-Mora ME, Ramos L. Los malestares masculinos: Narraciones de un grupo de varones adultos de la Ciudad de México. Salud Mental 2008;31:38I-339.

5. Rafful C, Medina-Mora ME, Borges G, Benjet C, Orozco R. Depression, gender and the treatment gap in Mexico. JAffect Disord 20I2; I38;35:I-II. 6. Medina-Mora ME, Rojas E, Borges G, Vázquez-Pérez, Fleiz C, Real

T. Comorbidity: Depression and substance abuse. En: Aguilar-Gaxiola S, Gullotta T, eds. Depression in Latinos. Assessment, treatment and prevention. New York: Springer, 2008.

7. Breslau J, Borges G, Hagar Y, Tancredi D, Gilman S. Immigration to the USA and risk for mood and anxiety disorders: variation by origin and age at immigration. Psychol Med 2009;39: I I 17-I I 27.

8. Medina-Mora ME, Borges G, Lara C, Ramos L, Zambrano J, Fleiz C. Prevalencia de sucesos violentos y de trastorno por estrés postraumático en la población mexicana. Salud Publica Mex 2005a; 47:8-22.

9. Medina-Mora ME, Borges G, Lara C, Benjet C, Blanco J, et al. Prevalence, service use, and demographic correlates of 12-month DSMIV psychiatric disorders in Mexico: results from the Mexican National Comorbidity Survey. Psychol Med 2005b; 35(12): 1773-1783.

10. Kessler R, Tat W, Demler O, Walters E. Prevalence, severity, and comorbidity of I2-month DSM-IV disorders in the National Comorbidity Survey Replication. Arch Gen Psychiatry 2005;62:617-627.

II. Feijó M, Kohn R, Mari J, Andrade L, Almeida-Filho N, Blay S, et al. La epidemiología de las enfermedades mentales en Brasil. En: Rodríguez J, Kohn R, Aguilar-Gaxiola S, eds. Epidemiología de los trastornos mentales en América Latina y el Caribe. Washington: Organización Panamericana de la Salud, 2009:79-89.

12. Vicente B, Kohn R, Saldivia S, Rioseci P. Las contribuciones de Chile a la investigación epidemiológica psiquiátrica. En: Rodríguez J, Kohn R, AguilarGaxiola S, eds. Epidemiología de los trastornos mentales en América Latina y el Caribe. Washington: Organización Panamericana de la Salud, 2009:|8-|3|.

13. Posada-Villa J, Aguilar-Gaxiola S, Deeb-Sossa N. La patología psiquiátrica en Colombia: Resultados del Estudio Nacional de Salud Mental, Colombia, 2003. En: Rodríguez J, Kohn R, Aguilar-Gaxiola S, eds. Epidemiología de los trastornos mentales en América Latina y el Caribe. Washington: Organización Panamericana de la Salud, 2009:64-78. 14. Lara MC, Medina-Mora ME, Borges G, Zambrano J. Social cost of mental disorders: disability and work days lost. Results from the Mexican Survey of Psychiatric Epidemiology. Salud Mental 2007;30:4-II.

15. González-Pier E, Gutiérrez-Delgado C, Stevens G, Barraza-Lloréns M, Porras-Condey R, Carvalho N, et al. Definición de prioridades para las intervenciones de salud en el Sistema de Protección Social en Salud de México. Salud Publica Mex 2007;49(Supl I):S37-S52.

16. Benjet C, Borges G, Blanco J, Rojas E, Fleiz E, Méndez E. et al. La Encuesta de Salud Mental en adolescentes de México. En: Rodríguez J, Kohn R, Aguilar-Gaxiola S, eds. Epidemiología de los trastornos mentales en América Latina y el Caribe. Washington: Organización Panamericana de la Salud, 2009;90-100.

17. Mejía-Arango S, Miguel-Jaimes A, Villa A, Ruiz-Arregui L, GutiérrezRobledo LM. Deterioro cognoscitivo y factores asociados en adultos mayores en México. Salud Publica Mex 2007;49 (Supl 4):475-48I. 18. Zúñiga E, Vega E. Envejecimiento de la población de México. Reto del siglo XXI. México: Consejo Nacional de Población, 2004:78.

19. Surkan PJ, Kennedy CE, Hurley KM, Black M. Maternal depression and early childhood growth in developing countries: systematic review and meta-analysis. Bull World Health Organ 201 I;287: 607-6I5.

20. Gaynes BN, Meltzer-Brody S, Lohr KN, Swinson T, Gartlehner G, Brody S, et al. Depression: prevalence, screening accuracy, and screening 
outcomes. Agency for Healthcare Research and Quality. Evidence Report No. 119. 2006 [consultado 2012 septiembre 18] Disponible en: www. ahrq.gov.

21. The Maternal Mental Health Work Group. House Bill 2666: Maternal Mental Health Work Group. Oregon, USA: Oregon Health Authority; 2010 [consultado 2012septiembre 18] Disponible en: http://www.oregon. gov/OHA/mentalhealth/child-mh-soc-in-plan-grp/reports/hb2666.pdf?ga=t. 22. Alvarado-Esquivel C, Sifuentes-Álvarez A, Estrada-Martínez S, SalasMartínez C, Hernández-Alvarado B, Ortiz-Rocha S, et al. Prevalencia de depresión posnatal en mujeres atendidas en hospitales públicos de Durango, México. Gac Med Mex 2010;146:I-9.

23. Antenatal and postnatal mental health. The nice guideline on clinical management and service guidance. The British Psychological Society. Great Britain: Alden Press; 2007. [consultado 2012 septiembre 20] Diponible en: http://www.nice.org.uk/nicemedia/pdf/CG45fullguideline.pdf.

24. Lara MA, Navarro C, Navarrete L. Outcome results of a psychoeducational intervention in pregnancy to prevent PPD: A randomized control trial. J Affecti Disord2010;122:109-1 17.

25. Bennett H, Einarson A, Taddio A, Koren G, Einarson, T. Depression during pregnancy. Overview of clinical factors. Clin Drug Invest 2004:24:157-179.

26. Lara MA, Le HN, Letechipia G, Hochhausen L. Prenatal depression in Latinas in the U.S. and Mexico. Matern Child Health J 2009; 13:567-76.

27. Davis WK, Hess GE, Hiss RG. Psychological correlates of survival in diabetes. Diabetes Care 1988; I I:538-545.

28. Word Federation for Mental Health. Mental health and chronic physical illnesses. The need for continued and integrated care. USA: World Federation for Mental Health, 2010

29. Caraveo J. Epidemiología psiquiátrica en México 1977-2000. En: Rodríguez J, Kohn R, Aguilar-Gaxiola S, eds. Epidemiología de los trastornos mentales en América Latina y el Caribe. Washington: Organización Panamericana de la Salud, 2009;132-144.

30. Bittner A, Goodwin RD, Wittchen H, Beesdo H, Höfle M, Lieb R. What characteristics of primary anxiety disorders predict subsequent major depressive disorder? J Clin Psychiat 2004;65:618-626.

31. Wang P, Angermeyer M, Borges G, Bruffaerts R, Tat Chiu W, Girolamo G, et al. Delay and failure in treatment seeking after first onset of mental disorders in the World Mental Health Survey Initiative. En: Kessler R, Ustun TB, eds. The WHO World Mental Health Surveys.
Global perspectives on the epidemiology of mental disorders. Cambridge University Press/World Health Organization, 2008;522-533.

32. Borges G, Medina-Mora ME, Wang P, Lara C, Berglund P, Walters E. Treatment and adequacy of treatment of mental disorders among respondents to the Mexico National Comorbidity Survey. Am J Psychiatry 2006;|63:137|-|378.

33. Berenzon, S, Saavedra N, Alanis S. Estrategias utilizadas por un grupo de mujeres mexicanas para cuidar su salud emocional. Autoatención y apoyo social. Salud Publica Mex 2009;51:474-48I.

34. Wagner F, González-Forteza C, Sánchez-García S, García-Peña C, Gallo J. Enfocando la depresión como problema de salud pública en México. Salud Mental 20I2; 35(I):3-II.

35. Chisholm D. Dollars, DALYs and decisions: economic aspects of the mental health system. Geneva: World Health Organization, 2006. 36. Lara-Muñoz MC, Robles-García R, Orozco R, Real T, Chisholm D, Medina-Mora ME. Estudio de costo-efectividad del tratamiento de la depresión en México. Salud Mental 2010;33:301-308.

37. Thase ME, Greenhouse JB, Frank E, ReynoldsCF, Pilkonis PA, Hurley K, et al. Treatment of major depression with psychotherapy or psychotherapy-pharmacotherapy combinations. Arch Gen Psychiatry 1997;54;1009-10I5.

38. Muñoz, RF, Ghosh Ippen C, Rao S, Le HL, Dwyer EV. Manual de terapia de grupo para el tratamiento cognitivo-conductual de la depresion: Aprendiendo a manejar su realidad personal. [consultado 2012 septiembre 26] Disponible en: http://www.medschool.ucsf.edu/latino/ cbtdspan.aspx

39. Páez F, Robles $R$, Chávez J. Eficacia de una intervención psicológica impartida por un pasante de medicina para disminuir la sintomatología depresiva y ansiosa en mujeres rurales del estado de Jalisco. Trabajo de investigación presentado en el Encuentro Nacional de Investigadores. Veracruz, México: Secretaría de Salud, 2002.

40. Lara M, Navarro C, Rubí N, Mondragón, L. Outcome of two levels of intervention in low-income women with depressive symptoms. Am J Orthopsychiatry 2003; 73(I): 35-43.

4I. Riveros A, Cortazar-Palapa J, Alcazar F, Sánchez Sosa JJ. Efectos de una intervención cognitivo conductual en la calidad de vida, ansiedad, depresión y condición médica de pacientes diabéticos e hipertensos esenciales. Int J Clin Health Psychol 2005;5:445-462. 